\title{
Pathological classification of hepatic inflammatory pseudotumor with respect to IgG4-related disease
}

\author{
Yoh Zen ${ }^{1,2}$, Takahiko Fujii ${ }^{1}$, Yasunori Sato ${ }^{1}$, Shinji Masuda ${ }^{3}$ and Yasuni Nakanuma ${ }^{1}$ \\ ${ }^{1}$ Department of Human Pathology, Graduate School of Medicine, Kanazawa University, Kanazawa, Japan; \\ ${ }^{2}$ Division of Pathology, Kanazawa University Hospital, Kanazawa, Japan and ${ }^{3}$ Department of Pathology, \\ Kouseiren Takaoka Hospital, Takaoka, Japan
}

\begin{abstract}
Recently, much attention has focused on IgG4-related disease, which is characterized by abundant IgG4positive plasma cell infiltration and high serum IgG4 levels. IgG4-related disease sometimes manifests as tumorous lesions, and its relationship to inflammatory pseudotumor has been suggested. In this study, we examined clinicopathological features of a total of 16 cases of hepatic inflammatory pseudotumor (11 men and 5 women with an average age of 67 years) with respect to IgG4-related disease. The tumors could be pathologically classified into two types: fibrohistiocytic (10 cases) and lymphoplasmacytic (6 cases). Fibrohistiocytic inflammatory pseudotumors were characterized by xanthogranulomatous inflammation, multinucleated giant cells, and neutrophilic infiltration, and mostly occurred in the peripheral hepatic parenchyma as mass-forming lesions. In contrast, lymphoplasmacytic inflammatory pseudotumors showed diffuse lymphoplasmacytic infiltration and prominent eosinophilic infiltration, and were all found around the hepatic hilum. In addition, venous occlusion with little inflammation and cholangitis without periductal fibrosis were frequently observed in the fibrohistiocytic type, whereas obliterative phlebitis and cholangitis with periductal fibrosis were common features of the lymphoplasmacytic type. Interestingly, IgG4-positive plasma cells were significantly more numerous in the lymphoplasmacytic than fibrohistiocytic type. However, two of the fibrohistiocytic inflammatory pseudotumors had relatively many IgG4-positive plasma cells. In conclusion, hepatic inflammatory pseudotumor could be classified into two types based on clinicopathological characteristics. The lymphoplasmacytic type is unique, and could belong to the so-called IgG4-related diseases. In contrast, the fibrohistiocytic type might still be a heterogeneous group of disorders. This latter type seems pathologically different from IgG4-related disease, although cases with relatively abundant IgG4-positive plasma cells should be differentiated from IgG4-related disease with secondary histopathologic modifications. Modern Pathology (2007) 20, 884-894; doi:10.1038/modpathol.3800836; published online 15 June 2007
\end{abstract}

Keywords: inflammatory myofibroblastic tumor; plasma cell granuloma; cholangitis; autoimmune pancreatitis; phlebitis

Inflammatory pseudotumors occur most commonly in the lung, and only occasionally in extrapulmonary organs including the liver. ${ }^{1,2}$ In a large series of 84 extrapulmonary inflammatory pseudotumors reported by Coffin et al, just 7 (8\%) were located in the liver. ${ }^{3}$ Inflammatory pseudotumors are characterized histologically by the proliferation of fibroblasts or myofibroblasts and inflammatory cell infiltration, irrespective of their organs of origin. ${ }^{1-5}$ The inflammatory cells mainly consist of polyclonal lympho-

Correspondence: Dr Y Nakanuma, MD, Department of Human Pathology, Kanazawa University Graduate School of Medicine, 13-1 Takaramachi, Kanazawa 920-8640, Japan.

E-mail: pbcpsc@kenroku.kanazawa-u.ac.jp

Received 19 March 2007; revised 18 May 2007; accepted 21 May 2007; published online 15 June 2007 cytes and plasma cells. Bacterial or viral infections and autoimmune reactions were suggested in the development of some inflammatory pseudotumors, although the etiology and pathogenesis of hepatic inflammatory pseudotumor have not been well documented and might differ among cases. ${ }^{6,7}$

Recently, a close relationship between IgG4related immune reactions and inflammatory pseudotumor was suggested. In 2001, Hamano et $a l^{8}$ reported that serum IgG4 levels were specifically elevated in patients with autoimmune pancreatitis compared to those with other inflammatory or neoplastic diseases of the pancreatobiliary system. Autoimmune pancreatitis is known to manifest a tumorous swelling of the pancreatic parenchyma, and has been referred to as pancreatic inflammatory pseudotumor in some reports. ${ }^{9}$ Pathologically, 
autoimmune pancreatitis is a lymphoplasmacytic sclerosing pancreatitis, characterized by severe lymphoplasmacytic infiltration, irregular fibrosis, obliterative phlebitis, acinar atrophy, a frequent association with sclerosing cholangitis, and the extension of inflammatory processes to peripancreatic adipose tissue. ${ }^{10,11}$ Abundant IgG4-positive plasma cells are easily found in inflamed areas by immunostaining, suggesting that IgG4 is not only a serum marker but also one of the pathogenetic factors for autoimmune pancreatitis. ${ }^{12,13}$

In 2004, we reported that hepatic inflammatory pseudotumor associated with sclerosing cholangitis had the exact same pathological characteristics as autoimmune pancreatitis. ${ }^{14}$ The cases showed severe lymphoplasmacytic infiltration by many IgG4positive plasma cells, obliterative phlebitis, and irregular fibrosis. We speculated that characteristic immune reactions related to IgG4 cause tumorous inflammation in the hepatobiliary and pancreatic systems. ${ }^{14}$ Interestingly, pulmonary and mammary inflammatory pseudotumors had similar pathological features including numerous IgG4-positive plasma cells. ${ }^{15,16}$ Those reports suggested a pathological and immunological resemblance between IgG4related disease and inflammatory pseudotumor. However, it is not clear whether or not all hepatic inflammatory pseudotumors are related to IgG4, or hepatic inflammatory pseudotumor can be histologically classified based on IgG4-positive cells.

In this study, we tried to categorize hepatic inflammatory pseudotumors into two types, and examined the clinicopathological characteristics of each type in comparison with IgG4-related disease.

\section{Materials and methods}

\section{Case Selection}

A total of 16 cases in which the original diagnosis was hepatic inflammatory pseudotumor were selected from the hepatobiliary disease files of the Division of Pathology, Kanazawa University Hospital and affiliated hospitals in Japan for the period between 1990 and 2005. All cases involved surgical resection (eight cases, lobectomy; five cases, segmentectomy; and 3 cases, partial resection). The patients were 11 men and 5 women with an average age of 67 years (range: 56-82). Five of the cases were used in our previous study. ${ }^{14}$ Hepatic inflammatory pseudotumor was defined as nodular lesions in the liver parenchyma or hepatic hilum, all of which consisted pathologically of fibroblastic or myofibroblastic proliferation intermixed with inflammatory cell infiltration without evident neoplastic features. All patients were radiologically diagnosed with 'a hepatic tumor' (eight cases, hilar cholangiocarcinoma; five cases, intrahepatic cholangiocarcinoma; two cases, malignant hepatic tumor of undetermined origin; one case, metastatic carcinoma). Any patient with clinically or radiologically diagnosed abscesses was excluded from the present study. No subjects had any preceding biliary diseases such as hepatolithiasis or liver flukes.

\section{Tissue Processing}

Surgically resected material was examined macroscopically after its immersion in formalin and cut in parallel at 5-mm intervals. Multiple sections (average: 4.7 specimens; range: 2-9) were obtained from each nodule. Formalin-fixed and paraffin-embedded sections were prepared and used for histopathological and immunohistochemical examination. Four-micrometer sections were cut for hematoxylin and eosin (H\&E), reticulin, elastica van Gieson (EVG), and immunohistochemical stainings, and for in situ hybridization.

\section{Classification of Bile Ducts}

The biliary tree is classified into the extrahepatic bile ducts (common hepatic duct and common bile duct), the gallbladder, the right and left hepatic ducts, and their branches. Intrahepatic large bile ducts correspond to the first to third branches of the right and left hepatic ducts, and the intrahepatic small bile ducts are composed of septal and interlobular bile ducts. ${ }^{17}$ Hepatic hilar ducts refer to the hepatic portion of the common hepatic duct, the right and left hepatic ducts, and the intrahepatic large bile ducts in this study.

\section{Immunohistochemistry}

Immunostaining of IgG, IgG4, $\alpha$ SMA, CD68, CD21, CD35, and anaplastic lymphoma kinase (ALK) was performed using the EnVision + system (Dako Cytomation, Glostrup, Denmark). Deparaffinized sections were microwaved in citrate buffer ( $\mathrm{pH}$ 6.0) (sections for IgG and CD68), ethylenediaminetetraacetic acid buffer ( $\mathrm{pH}$ 8.0) (sections for IgG4 and ALK), or Target Retrieval Solution (Dako Cytomation) (sections for CD21 and CD35) for 20 min. After the blocking of endogenous peroxidase and incubation in protein block solution (Dako Cytomation) for $20 \mathrm{~min}$, the deparaffinized sections were incubated overnight at $4^{\circ} \mathrm{C}$ with primary antibodies: anti-IgG (rabbit polyclonal, A0423, 1:200, Dako Cytomation), anti-IgG4 (mouse monoclonal, HP6025, 1:200, Zymed Laboratory, San Francisco, CA, USA), anti- $\alpha$ SMA (mouse monoclonal, 1A4, 1:200, Dako Cytomation), antiCD68 (mouse monoclonal, KP-1, 1:600, Dako Cytomation), anti-CD21 (mouse monoclonal, 1F8, 1:25, Dako Cytomation), anti-CD35 (mouse monoclonal, Ber-MAC-DRC, 1:25, Dako Cytomation), and antiALK (rabbit monoclonal, SP8, 1:50, Nichirei, Tokyo, Japan). The sections were then incubated at room temperature for $1 \mathrm{~h}$ with goat anti-mouse and antirabbit immunoglobulins conjugated to peroxidaselabeled dextran polymer (EnVision + ; Dako Cytoma- 
tion). The reaction products were developed by immersing the section in a 3,3'-diaminobenzidine tetrahydrochloride solution containing $0.03 \%$ hydrogen peroxide. Nuclei were lightly counterstained with hematoxylin. IgG- or IgG4-positive plasma cells were counted in five different high-power fields (hpf: $\times 10$ eyepiece and $\times 40$ lens) with intense inflammation, and the ratio (percentage) of IgG4-/IgG-positive plasma cells was calculated in each case. Positive controls used were tonsil (IgG, IgG4, CD68, CD21, and CD35), the muscle layer of the stomach ( $\alpha$ SMA), and lymph node with anaplastic lymphoma (ALK).

\section{In Situ Hybridization}

In situ hybridization of $\kappa$ - and $\lambda$-chains was performed by an autostainer (HX System Benchmark, Ventana Medical Systems, Tucson, AZ, USA), as per the manufacturer's instructions. Specific probes for $\kappa$ - and $\lambda$-chains were obtained from Ventana Medical Systems. Specimens of gastric marginal zone B-cell lymphoma (MALToma) and tonsillitis were used as positive and negative controls, respectively.

\section{Statistical Analysis}

Statistical analysis was performed using the MannWhitney $U$-test. A probability of $P<0.05$ was considered statistically significant.

\section{Results}

\section{Histological Classification of Hepatic Inflammatory Pseudotumors}

The sixteen hepatic inflammatory pseudotumors were classifiable into two types based on histological characteristics: fibrohistiocytic and lymphoplasmacytic. Ten tumors were classified as fibrohistiocytic, six as lymphoplasmacytic. Stromal fibrosis with fibroblastic proliferation and inflammatory cell infiltration were common findings in both types. The fibrohistiocytic type was defined as having abundant histiocytic infiltration involving foamy cells or multinucleated giant cells. The lymphoplasmacytic type was defined by an inflammatory process, mainly involving lymphoplasmacytic infiltration. Histiocytic infiltration was not conspicuous on H\&E staining in the lymphoplasmacytic tumors.

\section{Histological Characteristics of Hepatic Inflammatory Pseudotumor}

Next, we examined the following 11 pathological features in each case: xanthogranulomatous inflammation, multinucleated giant cells, discrete epithelioid granuloma, nodular eosinophilic deposition, neutrophilic infiltration ( $>10$ per $\mathrm{hpf}$ ), eosinophilic infiltration ( $>5$ per hpf), plasmacytic infiltration ( $>20$ per hpf), Russell bodies, entrapment of bile duct in nodules, venous changes, and cholangitis. As shown in Table 1, fibrohistiocytic-type inflammatory pseudotumors were commonly associated with xanthogranulomatous inflammation and multinucleated giant cells (Figure 1a-c). Some macrophages contained bile pigment. In addition, three of the fibrohistiocytic tumors had characteristic eosinophilic nodular deposits, which were surrounded by foamy macrophages or neutrophils (Figure 1d). Plump stromal cells, probably corresponding to active myofibroblasts, were commonly observed in the fibrohistiocytic type. In contrast, the lymphoplasmacytic type was histologically characterized by diffuse inflammatory cell infiltration, mainly by lymphocytes and plasma cells (Figure 2a and b). The inflammatory cells

Table 1 Comparison of histological features between hepatic inflammatory pseudotumors of fibrohistiocytic and lymphoplasmacytic types

\begin{tabular}{|c|c|c|c|}
\hline & Fibrohistiocytic type $(\mathrm{n}=10)$ & Lymphoplasmacytic type $(\mathrm{n}=6)$ & $\mathrm{P}$-value \\
\hline Xanthogranulomatous inflammation & $10(100 \%)$ & 0 & $<0.001$ \\
\hline Multinucleated giant cells & $5(50 \%)$ & 0 & 0.043 \\
\hline Epithelioid granuloma & $1(10 \%)$ & 0 & 0.439 \\
\hline Nodular eosinophilic deposition & $3(30 \%)$ & 0 & 0.150 \\
\hline Neutrophil infiltration ( $>10$ per hpf) & $6(60 \%)$ & 0 & 0.020 \\
\hline Eosinophil infiltration ( $>5$ per hpf) & $1(10 \%)$ & $4(67 \%)$ & 0.022 \\
\hline Plasma cell infiltration ( $>20$ per hpf) & $10(100 \%)$ & $6(100 \%)$ & Identical \\
\hline Russell bodies & $7(70 \%)$ & $6(100 \%)$ & 0.150 \\
\hline Involvement of bile ducts within nodule & $6(60 \%)$ & $6(100 \%)$ & 0.083 \\
\hline \multicolumn{4}{|l|}{ Venous changes } \\
\hline Obliterative phlebitis & $6(60 \%)$ & $6(100 \%)$ & 0.083 \\
\hline Venous occlusion & $4(40 \%)$ & 0 & 0.083 \\
\hline \multicolumn{4}{|l|}{ Cholangitis } \\
\hline Inflammatory cholangitis & $6(60 \%)$ & 0 & 0.020 \\
\hline Sclerosing cholangitis & 0 & $6(100 \%)$ & $<0.001$ \\
\hline
\end{tabular}

hpf, high-power fields; n, number of cases. 

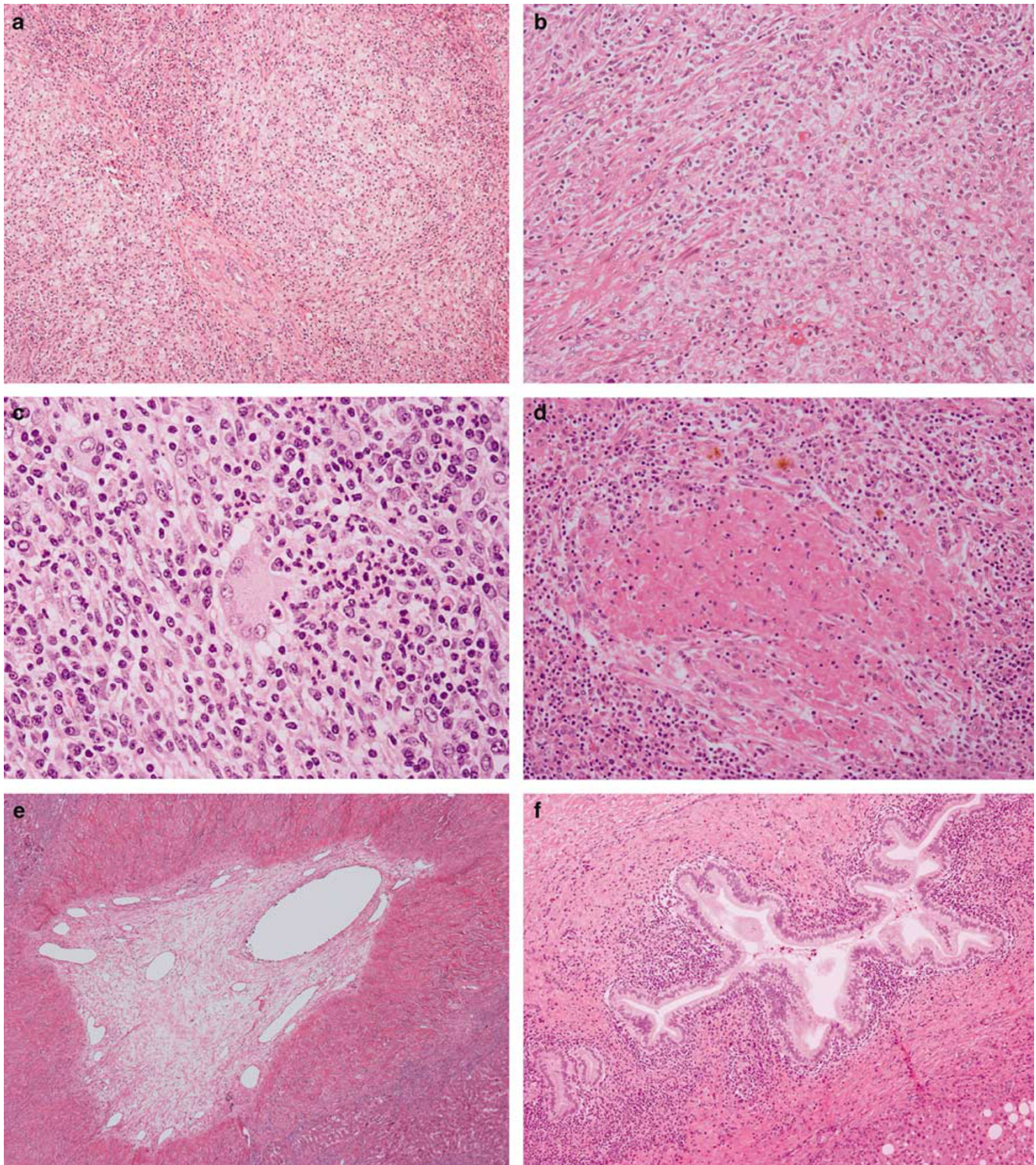

Figure 1 Histopathology of hepatic inflammatory pseudotumors (fibrohistiocytic type). (a) Many macrophages are observed in inflammatory pseudotumors. (b) Macrophages have a foamy cytoplasm. (c) This type of pseudotumor is associated with multinucleated giant cells and neutrophilic infiltration. (d) Nodular deposits of eosinophilic material are observed, and are surrounded by macrophages. (e) A hepatic vein tributary is incompletely occluded without evident inflammation (venous occlusion). Recanalization is found, suggesting an old thromboembolism. (f) The bile duct around this hepatic inflammatory pseudotumor showed cholangitis without sclerosis. All are H\&E staining; (a) $\times 100$; (b) $\times 200$; (c) $\times 400$; (d) $\times 200$; (e) $\times 40$; (f) $\times 100$.

infiltrated perineural spaces (Figure 2c). Lymphoplasmacytic tumors showed evident eosinophilic infiltration in four cases (67\%) and Russell bodies in all cases $(100 \%)$. Xanthogranulomatous inflammation was not observed in any cases of lymphoplasmacytic-type inflammatory pseudotumor.
Two types of venous occlusive change were observed in hepatic inflammatory pseudotumor. These lesions could be found in both hepatic-vein tributaries and portal-vein branches. Obliterative phlebitis, which is venous occlusion associated with inflammatory cell infiltration, was more com- 

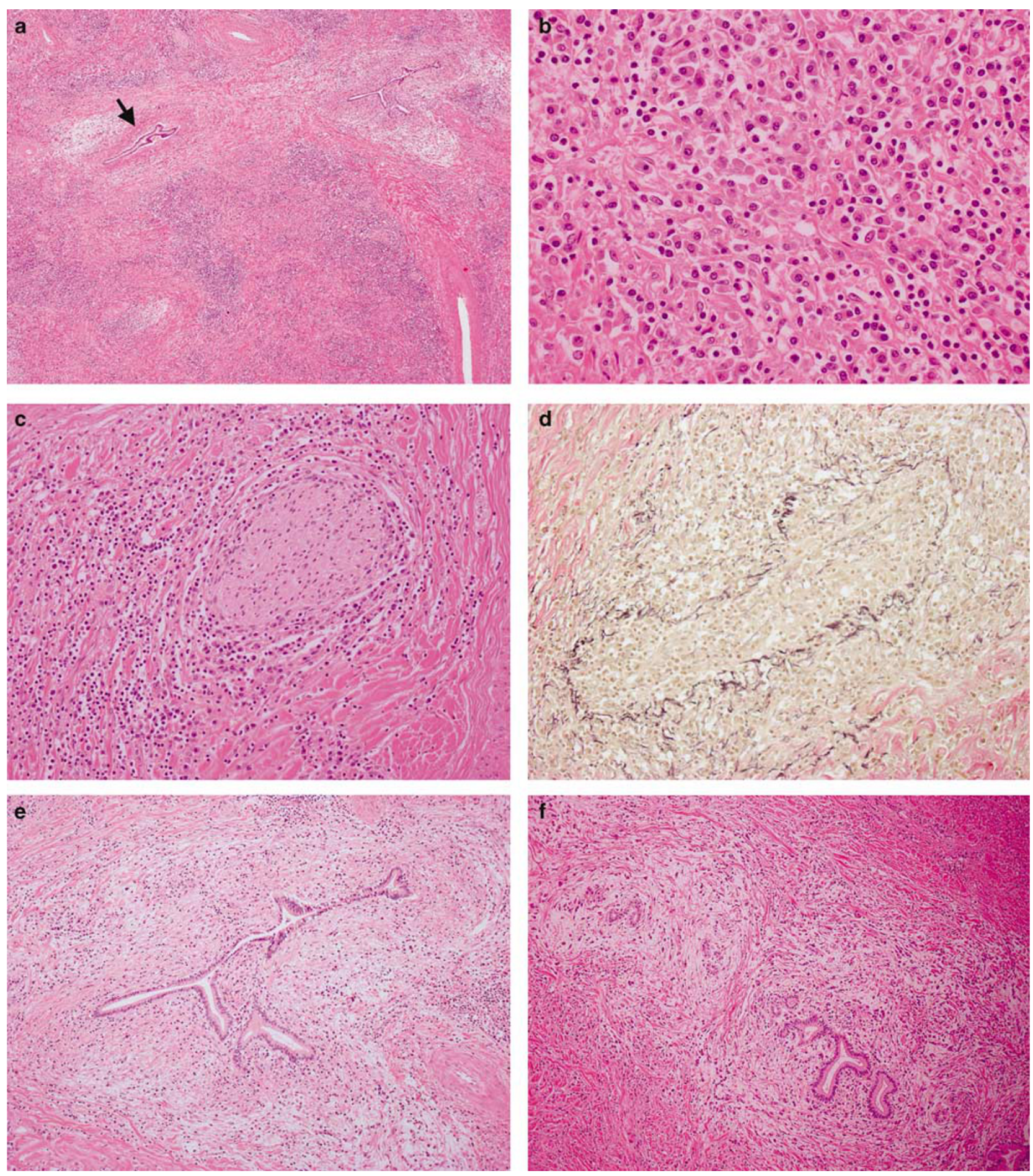

Figure 2 Histopathology of hepatic inflammatory pseudotumors (lymphoplasmacytic type). (a) Inflammatory pseudotumors consist of inflammatory cell infiltration and fibrosis. Bile ducts are entrapped in the nodule (arrow). (b) The inflammatory cells are mainly lymphocytes and plasma cells. (c) Inflammatory cells infiltrate perineural spaces. (d) Obliterative phlebitis is observed. (e) The bile duct around this hepatic inflammatory pseudotumor showed periductal inflammation and edematous fibrosis. (f) Sclerosing inflammation is also observed around peribiliary glands. (a-f) H\&E staining; (d) EVG; (a) $\times 40$; (b) $\times 400 ;(\mathbf{c}) \times 200 ;(\mathbf{d}) \times 200$; (e) $\times 100$; (f) $\times 100$.

mon in the lymphoplasmacytic type $(100 \%)$ than fibrohistiocytic type (60\%) (Figure 2d). Venous occlusion with little inflammation, probably reflecting an old thromboembolism, was identified only in the fibrohistiocytic type $(40 \%)$ (Figure 1e), and tended to be found in the larger vessels than obliterative phlebitis.

Bile ducts could be identified within nodules of hepatic inflammatory pseudotumor in both fibrohistiocytic and lymphoplasmacytic cases. Bile ducts 
in nodules were destroyed in the former, whereas the biliary epithelium was preserved, despite intense inflammation in the latter. In addition, cholangitis was identified in the bile ducts adjacent to each nodule of inflammatory pseudotumor in 12 cases. These cases could be classified as cholangitis with or without periductal edematous fibrosis. Inflammatory cholangitis, which is characterized by periductal lymphoplasmacytic infiltration without periductal concentric fibrosis, was observed only in the fibrohistiocytic type (Figure 1f). Sclerosing cholangitis showed periductal inflammatory cell infiltration with concentric edematous fibrosis, and this type of cholangitis was observed in all the patients with a lymphoplasmacytic tumor (Figure 2e). Sclerosing inflammation was also observed around peribiliary glands in the lymphoplasmacytic type (Figure 2f).

\section{Clinical Features of Hepatic Inflammatory Pseudotumor}

Average ages, man/woman ratios, tumor sizes, and tumor locations for each type of hepatic inflammatory pseudotumor are listed in Table 2. The fibrohistiocytic type occurred equally in both genders, whereas all cases of the lymphoplasmacytic type involved men. Locations of the tumor differed between the two types. The lymphoplasmacytic type of hepatic inflammatory pseudotumor was more commonly observed in the left lobe. Eight tumors $(80 \%)$ of the fibrohistiocytic type were located in the liver parenchyma showing massforming characteristics. In contrast, all hepatic inflammatory pseudotumors of the lymphoplasmacytic type were found in the hepatic hilum, and they distributed along hilar bile ducts. That is, fibrohistiocytic inflammatory pseudotumor macroscopically resembled mass forming-type intrahepatic cholangiocarcinoma, whereas lymphoplasmacytic inflammatory pseudotumor showed hilar lesions similar to periductal infiltrating-type hilar cholangiocarcinoma. Interestingly, the clinical presentation also differed between the two types. Eight patients $(80 \%)$ with fibrohistiocytic inflammatory pseudotumor had subjective symptoms such as fever, abdominal pain, and general malaise, whereas five patients $(83 \%)$ with the lymphoplasmacytic type were incidentally found to have liver dysfunction by routine laboratory testing.

Extrahepatobiliary lesions were observed in three cases. One patient with the fibrohistiocytic type had a carcinoma of Vater's ampulla. The hepatic inflammatory pseudotumor was surgically resected with suspicion of hepatic metastasis, and at the same time the Whipple procedure was used for the carcinoma of the Vater's ampulla. The carcinoma of the Vater's ampulla in this patient was a papillary adenocarcinoma involving the lower bile duct and pancreatic head. One case with lymphoplasmacytic inflammatory pseudotumor had a history of chronic hepatitis related to HCV infection. Interestingly, another patient with lymphoplasmacytic inflammatory pseudotumor underwent choledocho-jejunostomy for refractory pancreatitis, 6 years before the hepatic resection. This pancreatitis was diagnosed as autoimmune pancreatitis (lymphoplasmacytic sclerosing pancreatitis) by a pancreatic biopsy during choledocho-jejunostomy.

Table 2 Comparison of clinical characteristics between hepatic inflammatory pseudotumors of fibrohistiocytic and lymphoplasmacytic types

\begin{tabular}{|c|c|c|c|}
\hline & Fibrohistiocytic type $(\mathrm{n}=10)$ & Lymphoplasmacytic type $(\mathrm{n}=6)$ & $\mathrm{P}$-value \\
\hline Average age (range) & $68(59-82)$ & $67(56-79)$ & 0.586 \\
\hline Male/female ratio & $5 / 5$ & $6 / 0$ & 0.431 \\
\hline Nodular size (cm) & $2.8(1.5-4.5)$ & $3.4(2.0-4.8)$ & 0.231 \\
\hline Hepatic lobes & & & 0.197 \\
\hline Left lobe & $5(50 \%)$ & $5(83 \%)$ & \\
\hline Right lobe & $5(50 \%)$ & $1(17 \%)$ & \\
\hline Location & & & 0.003 \\
\hline Peripheral liver & $8(80 \%)$ & 0 & \\
\hline Hilar bile ducts & $2(20 \%)$ & $6(100 \%)$ & \\
\hline Shapes & & & 0.003 \\
\hline Mass-forming type & $8(80 \%)$ & 0 & \\
\hline Periductal infiltrating type & $2(20 \%)$ & $6(100 \%)$ & \\
\hline Clinical presentation & & & 0.017 \\
\hline Subjective symptoms & $8(80 \%)$ & 0 & \\
\hline Liver dysfunction by laboratory test & $2(20 \%)$ & $6(100 \%)$ & \\
\hline Extrahepatic lesion & Carcinoma of the Vater's ampulla (1) & $\begin{array}{l}\text { Autoimmune pancreatitis (1) } \\
\text { Chronic viral hepatitis (HCV) (1) }\end{array}$ & \\
\hline
\end{tabular}

n, number of cases; (1), one case having this extrahepatic lesion. 


\section{Immunohistochemistry and In Situ Hybridization of Hepatic Inflammatory Pseudotumor}

Histiocytes were not conspicuous in the sections of lymphoplasmacytic inflammatory pseudotumor subjected to H\&E staining, although relatively large numbers of CD68-positive cells were observed in all cases of hepatic inflammatory pseudotumor (Figure 3 ). In the fibrohistiocytic type, CD68-positive histiocyts were distributed diffusely, and also accumulated in a nodular fashion (Figure 3). CD68-positive cells were foamy shaped in the fibrohistiocytic type, whereas they were dendritic or spindle in shape in the lymphoplasmacytic type (insets in Figure 3). The expression of aSMA was observed in many stromal cells in fibrohistiocytic inflammatory pseudotumors (Figure 3). However, few stromal cells were positive for $\alpha \mathrm{SMA}$ in the lymphoplasmacytic tumors (Figure 3). Two follicular dendritic cell
(FDC) markers (CD21 and CD35) were detected in FDCs of lymphoid follicles, but not in any of the stromal cells in the hepatic inflammatory pseudotumors. These results suggested that the hepatic inflammatory pseudotumors examined in this study were different from the FDC tumors resembling inflammatory pseudotumor reported by Cheuk et $a .^{18}$ Immunostaining of ALK was negative in stromal cells and inflammatory cells, and in situ hybridization of $\kappa$ - and $\lambda$-chains revealed the polyclonal nature of lymphocytes and plasma cells in all cases of hepatic inflammatory pseudotumor.

\section{Immunostaining of IgG and IgG4 in Hepatic Inflammatory Pseudotumor}

Immunostaining of IgG revealed numerous IgGpositive plasma cells in all cases of hepatic

\section{Fibohistiocytic type}
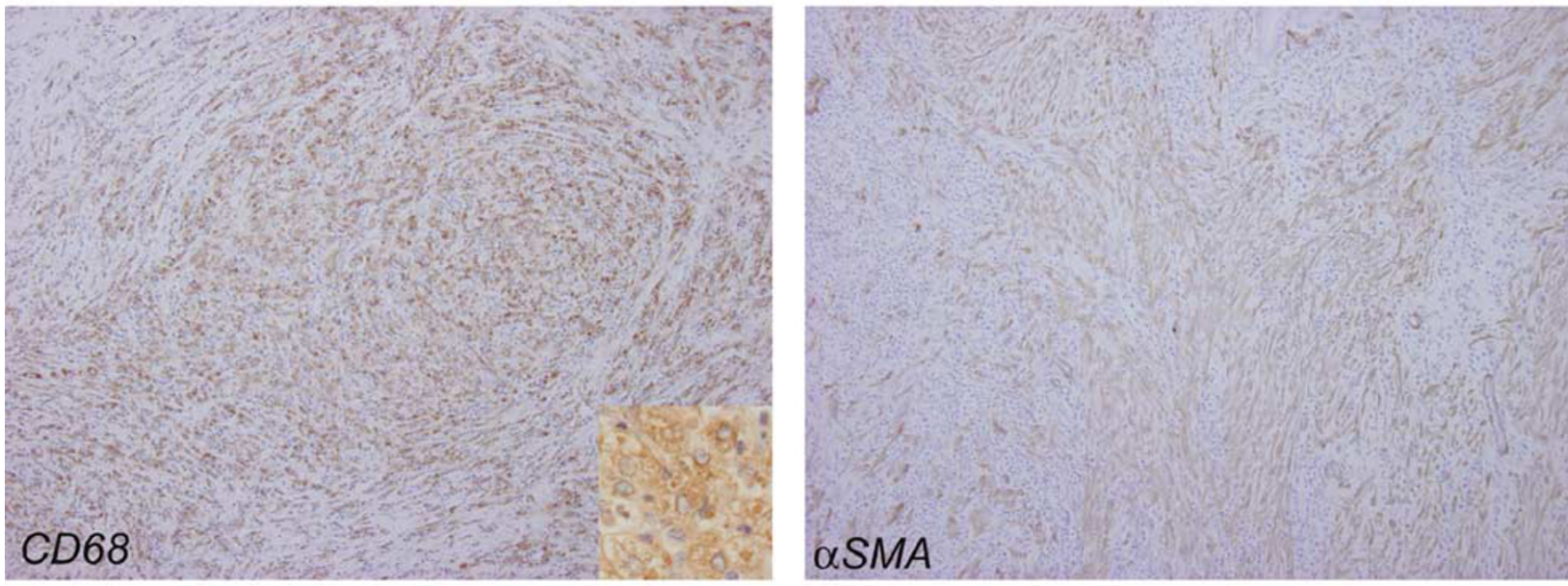

\section{Lymphoplasmacytic type}
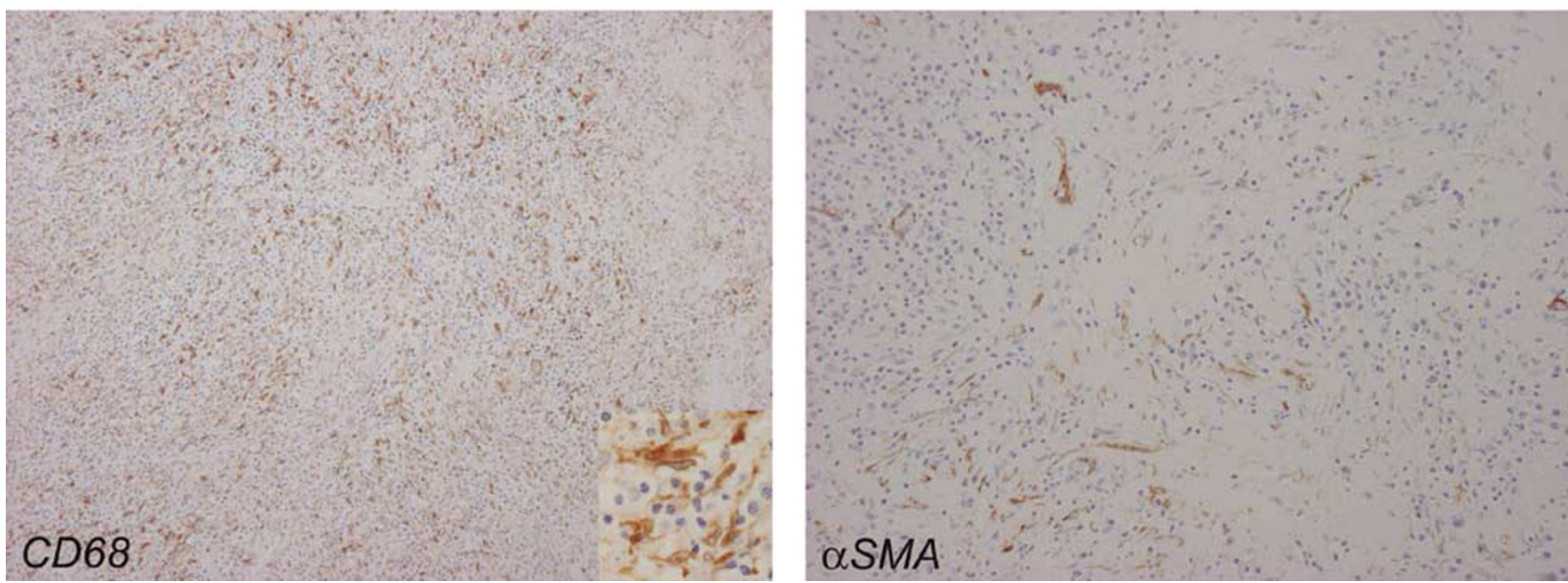

Figure 3 Immunostaining of CD68 and $\alpha$ SMA in hepatic inflammatory pseudotumors. Many CD68-positive cells are observed in both the fibrohistiocytic and lymphoplasmacytic types. CD68-positive macrophages show a nodular accumulation in the fibrohistiocytic type. CD68-positive cells are foamy in fibrohistiocytic inflammatory pseudotumors and spindle or dendritic shaped in lymphoplasmacytic inflammatory pseudotumors (insets). In the fibrohistiocytic type, many stromal cells express $\alpha$ SMA. In contrast, only a few spindle cells are positive for $\alpha$ SMA in the lymphoplasmacytic type. All images, $\times 100$; insets, $\times 400$. 
inflammatory pseudotumor, irrespective of type (Figure 4). Many IgG4-positive plasma cells were spread diffusely in all cases of the lymphoplasmacytic type, whereas seven cases of the fibrohistiocytic type had only a few IgG4-positive plasma cells (Figure 4). Numbers of IgG- or IgG4-positive plasma cells in each case are shown in Figure 5. The number of IgG-positive plasma cells was great in the lymphoplasmacytic than fibrohistiocytic type, although the difference was not significant. The number of IgG4-positive plasma cells was significantly larger in the lymphoplasmacytic than fibrohistiocytic type. From the ratio of IgG4-/IgG-positive plasma cells, lymphoplasmacytic and fibrohistiocytic inflammatory pseudotumors were clearly separated into high- and low-ratio groups, respectively (Figure 5).

Interestingly, two of the fibrohistiocytic tumors had relatively high IgG4-/IgG-positive plasma cell ratios (43.7 and $36.7 \%$ ). The hepatic inflammatory pseudotumor in these two cases involved xanthogranulomatous inflammation, multinucleated giant cells, evident neutrophilic infiltration, nodular eosinophilic deposition, and inflammatory cholangitis (Figure 6). These histological features corresponded to the fibrohistiocytic type. Interestingly, one of these patients had a much larger number of multinuclear giant cells than the other patients with fibrohistiocytic inflammatory pseudotumors (Figure 6).

\section{Discussion}

The main findings of this study are as follows: (1) hepatic inflammatory pseudotumor could be classified into two groups based on histological features; (2) the two types differed not only in pathological features, but also in clinical features such as clinical presentation, locations, and shapes; (3) the lymphoplasmacytic type showed diffuse lymphoplasmacytic

\section{Fibohistiocytic type}
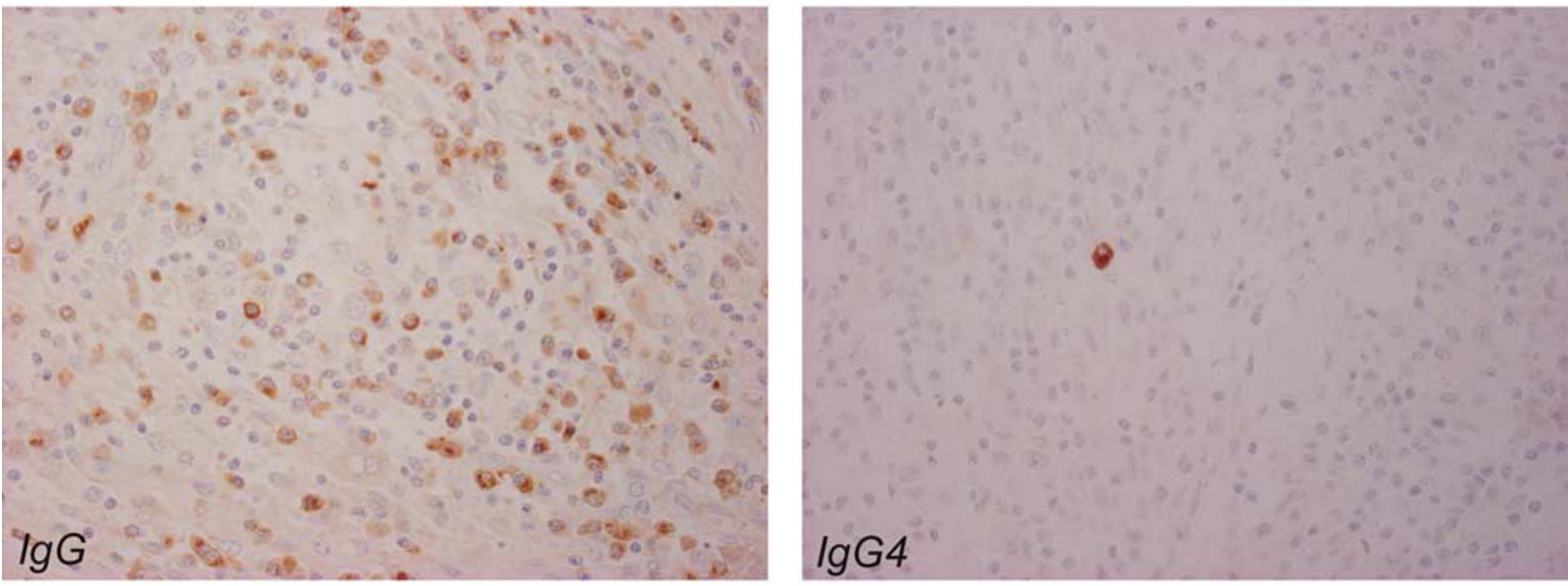

\section{Lymphoplasmacytic type}
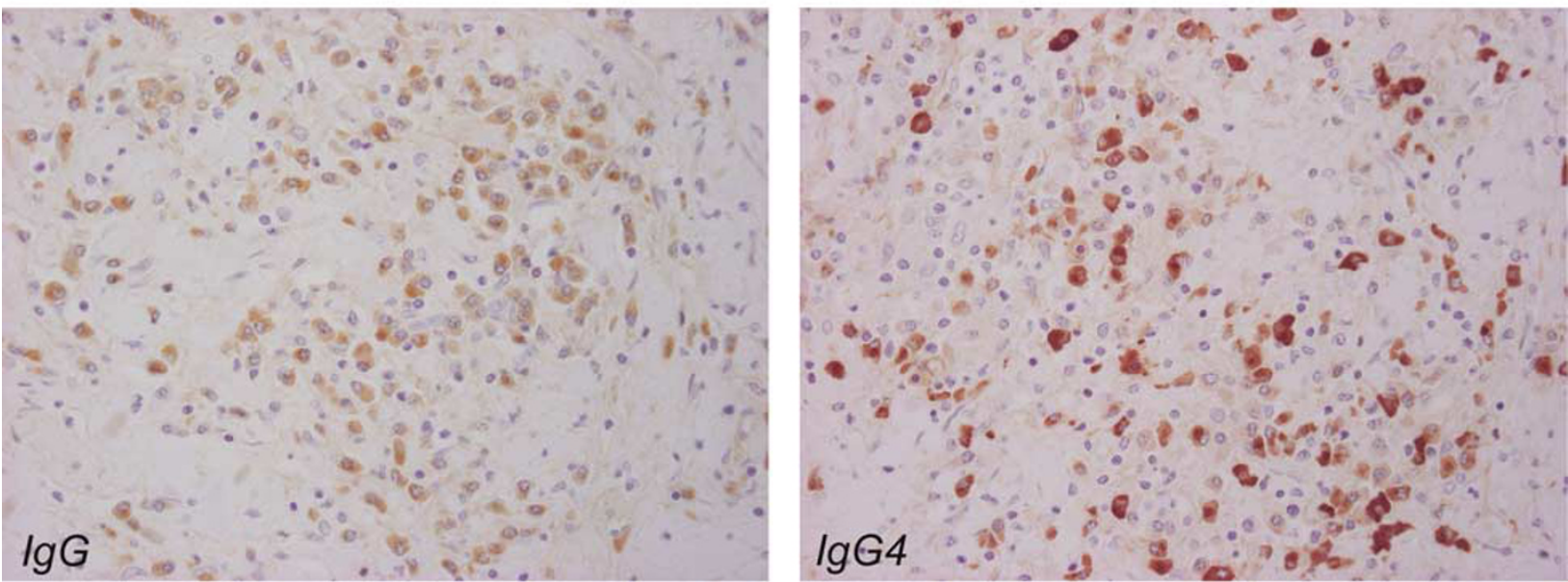

Figure 4 Immunostaining of IgG and IgG4 in hepatic inflammatory pseudotumors. IgG-positive plasma cells are abundantly observed in both the fibrohistiocytic and lymphoplasmacytic types. IgG4-positive plasma cells are scarce in the fibrohistiocytic type, whereas they are widespread in the lymphoplasmacytic type. All images, $\times 400$. 

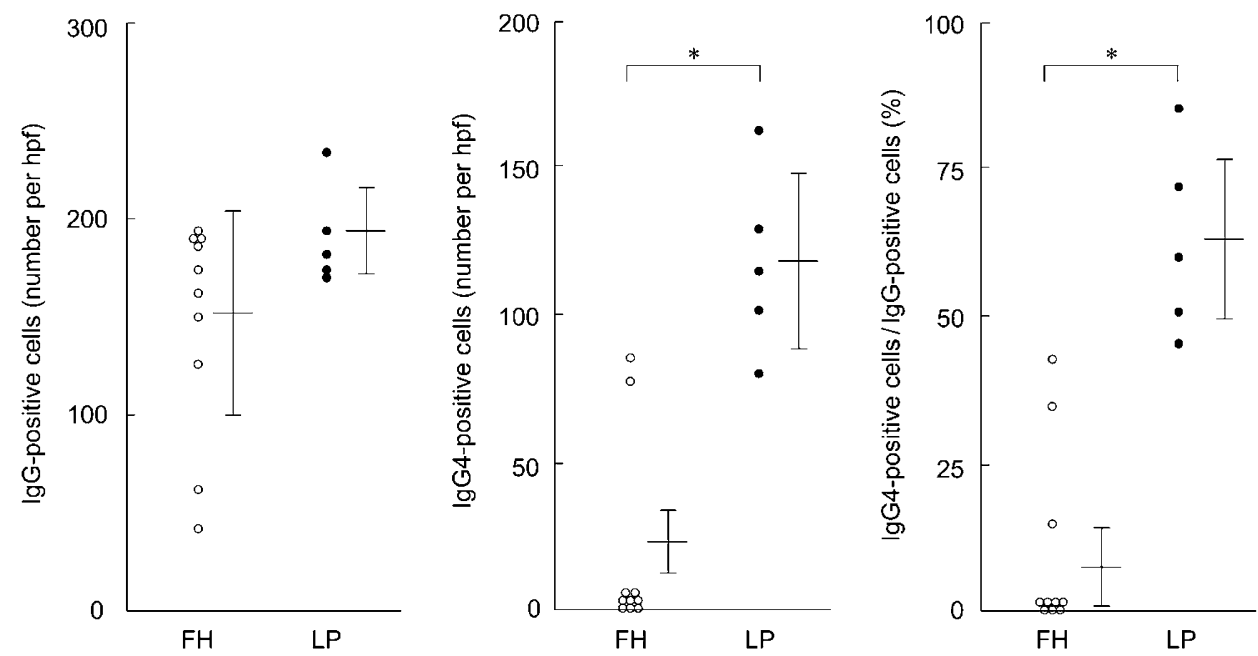

Figure 5 IgG- and IgG4-positive plasma cells in hepatic inflammatory pseudotumors. The number of IgG-positive plasma cells is relatively high in the lymphoplasmacytic type (LP) compared to the fibrohistiocytic type (FH) $(P=0.254)$. The number of IgG4-positive plasma cells and its ratio to IgG-positive plasma cells are significantly greater in the lymphoplasmacytic type than fibrohistiocytic type $(P=0.001)$. Bar, average \pm s.d.; ${ }^{*} P<0.05$.
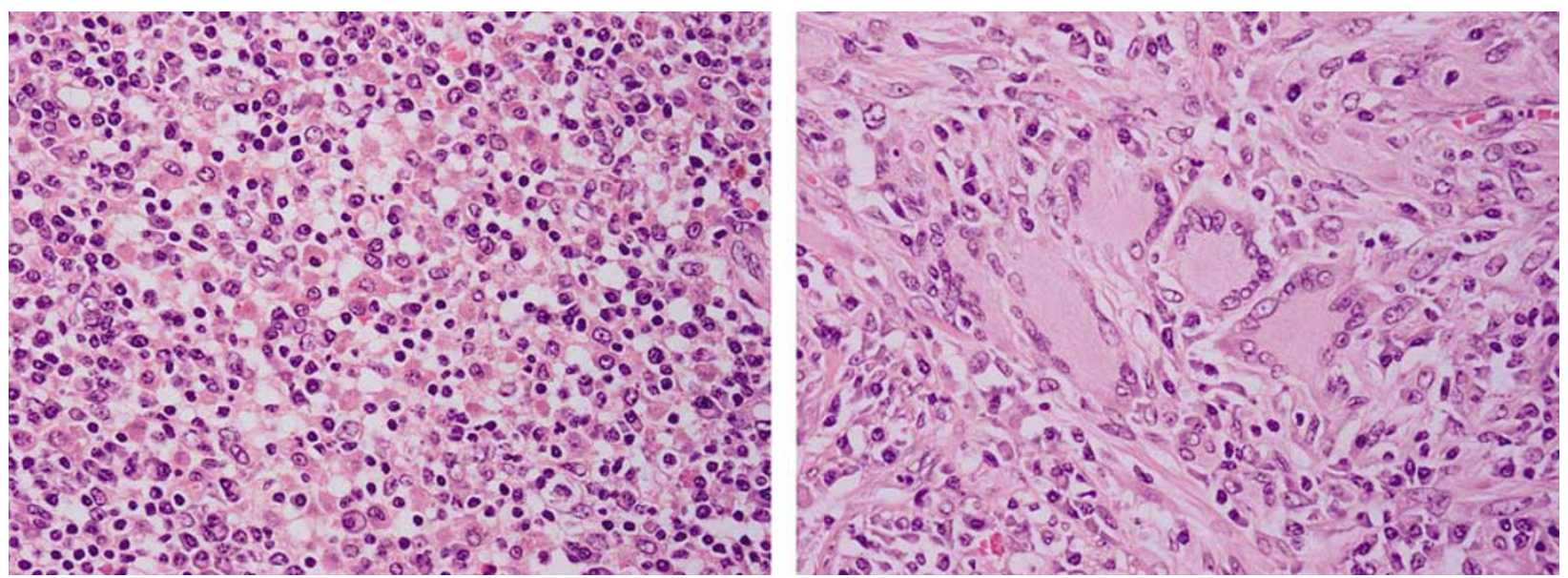

Figure 6 Histopathology of hepatic inflammatory pseudotumors (fibrohistiocytic type) showing a high IgG4-/IgG-positive cell ratio. This hepatic inflammatory pseudotumor consists of xanthogranulomatous inflammation and many multinucleated giant cells. Such histological features correspond to the fibrohistiocytic type. All images, $\times 400$.

infiltration with numerous IgG4-positive plasma cells, and had histological features similar to IgG4related diseases such as autoimmune pancreatitis (lymphoplasmacytic sclerosing pancreatitis); (4) two hepatic inflammatory pseudotumors were associated with relatively large numbers of IgG4-positive plasma cells, although in terms of histological characteristics, they seemed to belong to the fibrohistiocytic type, but not the lymphoplasmacytic type or IgG4-related disease.

Inflammatory pseudotumor is also known as xanthofibroma and inflammatory myofibroblastic tumor, suggesting it to be a heterogeneous disease entity occurring in various organs. ${ }^{19}$ Inflammatory pseudotumors are sometimes classified into subtypes such as fibrohistiocytic type, xanthogranulomatous type, spindle cell type, and plasma cell granuloma, although there is still no uniformly accepted classification system. In this study, hepatic inflammatory pseudotumors were largely classified into two categories based on their major histologies: fibrohistiocytic and lymphoplasmacytic types. The former was characterized by histiocytic infiltration, such as xanthogranulomatous inflammation and multinucleated giant cells, whereas the latter showed inflammatory processes, mainly infiltration by lymphocytes and plasma cells. According to descriptions of hepatic inflammatory pseudotumors in previous reports, the lymphoplasmacytic type seems to correspond to so-called plasma cell granuloma. ${ }^{19,20}$ In addition, it was found that the two types of hepatic inflammatory pseudotumor had different clinicopathological features, including immunohistochemistry, clinical presentation, and 
the location of the tumors, suggesting the validity of this classification system.

Interestingly, IgG4-positive plasma cells were distributed in a diffuse manner in all of the lymphoplasmacytic cases. Recently, much attention has been drawn to IgG4-related disease, which is a disease entity first proposed with regard to autoimmune pancreatitis., ${ }^{8,21,22}$ IgG4-related disease can occur in various organs such as the salivary gland (chronic sclerosing sialadenitis), ${ }^{23}$ lung (inflammatory pseudotumor or interstitial pneumonia), ${ }^{16,24}$ and retroperitoneum (retroperitoneal fibrosis). ${ }^{12,25}$ It has histological characteristics like lymphoplasmacytic infiltration, irregular fibrosis, eosinophilic infiltration, and abundant IgG4-positive plasma cells. Lymphoplasmacytic inflammatory pseudotumors shared all of these histological features of IgG4-related disease. Previously, we proposed that IgG4-related disease frequently occurs in the hepatobiliary system presenting as sclerosing cholangitis with or without hepatic inflammatory pseudotumor. ${ }^{14}$ Lymphoplasmacytic inflammatory pseudotumors always occurred around the hepatic hilum, especially around the hilar bile ducts. The bile ducts adjacent or within inflammatory pseudotumors showed cholangitis with periductal concentric and edematous fibrosis, resembling IgG4-related sclerosing cholangitis. All of these findings strongly suggest that the lymphoplasmacytic form of hepatic inflammatory pseudotumor itself is a unique disease belonging to IgG4-related diseases.

IgG4-related diseases, including autoimmune pancreatitis, have been proposed as an autoimmune disorder. ${ }^{26,27}$ However, we recently examined in situ the production of cytokines in autoimmune pancreatitis and IgG4-related sclerosing cholangitis, and revealed that Th2 (IL-4, IL-5 and IL-13) and regulatory cytokines (IL-10 and TGF- $\beta$ ) were significantly expressed in IgG4-related disease. ${ }^{28}$ An immune reaction predominantly mediated by Th2 and regulatory cytokines seems unusual for classical autoimmune diseases, and rather, resembles an allergic immune response. In addition, IL-10 and TGF- $\beta$ are cytokines involved in IgG4 class switching and fibroplasia, respectively. ${ }^{29-31}$ We speculated that a hyperimmune reaction mediated by IL-10 and TGF- $\beta$ contributes to the characteristic pathological features of IgG4-related disease, like IgG4-positive plasma cell infiltration and fibrosis. This scenario could also work in lymphoplasmacytic-type hepatic inflammatory pseudotumor, although further studies are needed to resolve this issue.

In contrast, the etiopathogenesis of fibrohistiocytic-type hepatic inflammatory pseudotumor remains only speculative. This type showed xanthogranulomatous inflammation with multinucleated giant cells and neutrophils. Interestingly, there were many $\alpha$ SMA-positive myofibroblastic cells within the lesion, suggesting an ongoing fibrous process. Within the lesion, bile ducts were disrupted and venous branches were occluded, implicating de- structive inflammatory processes in the pathogenesis of this type of hepatic inflammatory pseudotumor. Cholangitis with periductal inflammation adjacent to nodules of inflammatory pseudotumor may be secondary to the inflammatory processes. This type could be the end stage of heterogeneous and destructive inflammatory processes in the liver, and heterogeneous etiologies may be responsible for this hepatic inflammatory pseudotumor. Interestingly, two of the fibrohistiocytic tumors had relatively large numbers of IgG4-positive plasma cells, although the other histological features of these two lesions were quite different from those of IgG4related disease. We speculate that the two cases were pathologically different from IgG4-related disease, although it might be possible that they originally involved IgG4-related lymphoplasmacytic inflammatory pseudotumor or sclerosing cholangitis, and secondary changes such as leakage of bile developed.

Inflammatory myofibroblastic tumor, which is also occasionally included in so-called hepatic inflammatory pseudotumor, ${ }^{19}$ has features suggestive of a neoplastic process. ${ }^{32}$ Myofibroblastic cells in this tumor usually show nuclear atypia, and the expression of ALK in myofibroblastic spindle cells is another characteristic, especially in children or young adults. ${ }^{32,33}$ There has been only one case of hepatic inflammatory myofibroblastic tumor expressing ALK reported in the literature. ${ }^{32}$ In the present study, no nuclear atypia was evident, and ALK was not detected immunohistochemically in any of the cases examined, suggesting that the lymphoplasmacytic and fibrohistiocytic types of hepatic inflammatory pseudotumor did not fit into the category of inflammatory myofibroblastic tumor.

In conclusion, we proposed a new system for pathological classification of hepatic inflammatory pseudotumors into lymphoplasmacytic and fibrohistiocytic types. The two types differed in their pathological characteristics and clinical features. Notably, the lymphoplasmacytic inflammatory pseudotumor seems to be a unique disease and to correspond to IgG4-related disease, whereas the fibrohistiocytic type might be a heterogeneous group of disorders.

\section{Acknowledgement}

We thank Dr Tsuneyama (Toyama University, Toyama, Japan) for their collaboration in providing histological specimens.

\section{References}

1 Shek TW, Ng IO, Chan KW. Inflammatory pseudotumor of the liver. Report of four cases and review of the literature. Am J Surg Pathol 1993;17:231-238. 
2 Horiuchi R, Uchida T, Kojima T, et al. Inflammatory pseudotumor of the liver. Clinicopathologic study and review of the literature. Cancer 1990;65:1583-1590.

3 Coffin CM, Humphrey PA, Dehner LP. Extrapulmonary inflammatory myofibroblastic tumor: a clinical and pathological survey. Semin Diagn Pathol 1998;15: 85-101.

4 Anthony PP. Inflammatory pseudotumour (plasma cell granuloma) of lung, liver and other organs. Histopathology 1993;23:501-503.

5 Nakanuma Y, Tsuneyama K, Masuda S, et al. Hepatic inflammatory pseudotumor associated with chronic cholangitis: report of three cases. Hum Pathol 1994;25:86-91.

6 Mergan F, Jaubert F, Sauvat F, et al. Inflammatory myofibroblastic tumor in children: clinical review with anaplastic lymphoma kinase, Epstein-Barr virus, and human herpesvirus 8 detection analysis. J Pediatr Surg 2005;40:1581-1586.

7 Priebe-Richter C, Ivanyi P, Buer J, et al. Inflammatory pseudotumor of the lung following invasive aspergillosis in a patient with chronic graft-vs host disease. Eur J Haematol 2005;75:68-72.

8 Hamano H, Kawa S, Horiuchi A, et al. High serum IgG4 concentrations in patients with sclerosing pancreatitis. N Engl J Med 2001;344:732-738.

9 Adsay NV, Basturk O, Klimstra DS, et al. Pancreatic pseudotumors: non-neoplastic solid lesions of the pancreas that clinically mimic pancreas cancer. Semin Diagn Pathol 2004;21:260-267.

10 Kawaguchi K, Koike M, Tsuruta K, et al. Lymphoplasmacytic sclerosing pancreatitis with cholangitis: a variant of primary sclerosing cholangitis extensively involving pancreas. Hum Pathol 1991;22:387-395.

11 Notohara K, Burgart LJ, Yadav D, et al. Idiopathic chronic pancreatitis with periductal lymphoplasmacytic infiltration: clinicopathologic features of 35 cases. Am J Surg Pathol 2003;27:1119-1127.

12 Hamano H, Kawa S, Ochi Y, et al. Hydronephrosis associated with retroperitoneal fibrosis and sclerosing pancreatitis. Lancet 2002;359:1403-1404.

13 Deshpande V, Chicano S, Finkelberg D, et al. Autoimmune pancreatitis: a systemic immune complex mediated disease. Am J Surg Pathol 2006;30: 1537-1545.

14 Zen Y, Harada K, Sasaki M, et al. IgG4-related sclerosing cholangitis with and without hepatic inflammatory pseudotumor, and sclerosing pancreatitis-associated sclerosing cholangitis: do they belong to a spectrum of sclerosing pancreatitis? Am J Surg Pathol 2004;28:1193-1203.

15 Zen Y, Kasahara Y, Horita K, et al. Inflammatory pseudotumor of the breast in a patient with a high serum IgG4 level: histologic similarity to sclerosing pancreatitis. Am J Surg Pathol 2005;29:275-278.

16 Zen Y, Kitagawa S, Minato $\mathrm{H}$, et al. IgG4-positive plasma cells in inflammatory pseudotumor (plasma cell granuloma) of the lung. Hum Pathol 2005;36: 710-717.

17 Nakanuma Y, Hoso M, Sanzen T, et al. Microstructure and development of the normal and pathologic biliary tract in humans, including blood supply. Microsc Res Tech 1997;38:552-570.
18 Cheuk W, Chan JK, Shek TW, et al. Inflammatory pseudotumor-like follicular dendritic cell tumor: a distinctive low-grade malignant intra-abdominal neoplasm with consistent Epstein-Barr virus association. Am J Surg Pathol 2001;25:721-731.

19 Travis WD, Colby TV, Koss MN, et al. Miscellaneous diseases of uncertain etiology. In: King DW (ed). Atlas of Nontumor Pathology. Non-Neoplastic Disorders of the Lower Respiratory Tract, 1st edn. American Registry of Pathology and Armed Forced Institute of Pathology: Washington, DC, 2002, pp 857-900.

20 Anthony PP. Inflammatory pseudotumour (plasma cell granuloma) of lung, liver and other organs. Histopathology 1993;23:501-503.

21 Neild GH, Rodriguez-Justo M, Wall C, et al. Hyper-IgG4 disease: report and characterisation of a new disease. BMC Med 2006;4:23.

22 Kamisawa $\mathrm{T}$, Funata N, Hayashi $\mathrm{Y}$, et al. Close relationship between autoimmune pancreatitis and multifocal fibrosclerosis. Gut 2003;52:683-687.

23 Kitagawa S, Zen Y, Harada K, et al. Abundant IgG4positive plasma cell infiltration characterizes chronic sclerosing sialadenitis (Küttner's tumor). Am J Surg Pathol 2005;29:783-791.

24 Taniguchi T, Ko M, Seko S, et al. Interstitial pneumonia associated with autoimmune pancreatitis. Gut 2004;53:770.

25 Zen Y, Sawazaki A, Miyayama S, et al. A case of retroperitoneal and mediastinal fibrosis exhibiting elevated levels of IgG4 in the absence of sclerosing pancreatitis (autoimmune pancreatitis). Hum Pathol 2006;37:239-243.

26 Asada M, Nishio A, Uchida K, et al. Identification of a novel autoantibody against pancreatic secretory trypsin inhibitor in patients with autoimmune pancreatitis. Pancreas 2006;33:20-26.

27 Aoki S, Nakazawa T, Ohara $\mathrm{H}$, et al. Immunohistochemical study of autoimmune pancreatitis using antiIgG4 antibody and patients' sera. Histopathology 2005;47:147-158.

28 Zen Y, Fujii T, Harada K, et al. Th2 and regulatory immune reactions are increased in IgG4-related sclerosing pancreatitis and cholangitis. Hepatology 2007;45:1538-1546.

29 Nouri-Aria KT, Wachholz PA, Francis JN, et al. Grass pollen immunotherapy induces mucosal and peripheral IL-10 responses and blocking IgG activity. J Immunol 2004;172:3252-3259.

30 Jeannin P, Lecoanet S, Delneste Y, et al. IgE vs IgG4 production can be differentially regulated by IL-10. J Immunol 1998;160:3555-3561.

31 Verrecchia F, Mauviel A, Farge D. Transforming growth factor-beta signaling through the Smad proteins: role in systemic sclerosis. Autoimmun Rev 2006;5:563-569.

32 Chan JK, Cheuk W, Shimizu M. Anaplastic lymphoma kinase expression in inflammatory pseudotumors. Am J Surg Pathol 2001;25:761-768.

33 Freeman A, Geddes N, Munson P, et al. Anaplastic lymphoma kinase (ALK 1) staining and molecular analysis in inflammatory myofibroblastic tumours of the bladder: a preliminary clinicopathological study of nine cases and review of the literature. Mod Pathol 2004;17:765-771. 